\title{
Weighted Least-squares Color Digital Halftoning
}

\author{
Zifen $\mathrm{He}^{1, \mathrm{a}}$, Yinhui Zhang ${ }^{1, \mathrm{~b}^{*}}$ \\ ${ }^{1}$ Kunming University of Science and Technology, Faculty of mechanical and electrical engineering, \\ Kunming, 650500, China \\ azyhhzf1998@163.com, ${ }^{\text {b }}$ yinhui_z@163.com
}

Keywords: Color halftoning; Human vision system(HVS);Weighted least-squares

\begin{abstract}
In this paper, we propose a weighted least-squares based color halftoning model from human vision system(HVS) model and an efficient iterative strategy using color image statistical information. The statistics of the mean and variance of the color pixel of each clustering are calculated. The energy function is constructed using the weighted least squares method, which the reciprocal of the variance of the segmented regions are referred to as the weighting operator. Starting with an initial halftone.The analysis and simulation results show that the proposed algorithm produces better color halftone image quality. A performance measure for halftone images is used to evaluate our algorithm. The value of MSEv and PSNR for the partitions regions that the proposed algorithm achieves consistently better values of MSEv and PSNR than the model-based color halftoning algorithm.
\end{abstract}

\section{Introduction}

The goal of digital color halftoning is to create the perception of a continuous-tone color image using the limited spatio-colorful discriminability of the human visual system. The algorithm for digital black and white halftoning methods can be categorized into three classes, which included error diffusion[1,2], dithering with blue noise[3], and direct binary search[4]. Color Halftoning is a method for creating the illusion of continuous tone output with a low-bit device. Effective digital color halftoning can substantially improve the quality of rendered images at minimal cost. Flohr et al. used the total squared error in a luminance/chrominance-based space as the metric for their model-based color halftoning algorithm[5] . A Ufuk exploited the differences in how the human viewers respond to luminance and chrominance information and use the total squared error[6].

\section{Image Segmentation Using Fuzzy c-means Clustering}

In fuzzy clustering, every point has a degree of belonging to clusters, as in fuzzy logic, rather than belonging completely to just one cluster. Thus, points on the edge of a cluster, may be in the cluster to a lesser degree than points in the center of cluster. An overview and comparison of different fuzzy clustering algorithms is available.[7]

Any point $x$ has a set of coefficients giving the degree of being in the $k$ th cluster $w_{k}(x)$. With fuzzy c-means, the centroid of a cluster is the mean of all points, weighted by their degree of belonging to the cluster:

$$
c_{k}=\frac{\sum_{x} w_{k}(x)^{m} x}{\sum_{x} w_{k}(x)^{m}}
$$

The degree of belonging, $w_{k}(x)$, is related inversely to the distance from $x$ to the cluster center as calculated on the previous pass. It also depends on a parameter $m$ that controls how much weight is given to the closest center. The fuzzy c-means algorithm is very similar to the k-means algorithm by choose a number of clusters[8]. 


\section{Weighted Least-squares Color Halftoning}

In this paper, we use the Flohr HVS model to design an efficient color halftoning algorithm. The luminance spatial frequency response model $H_{Y_{y}}(\bar{u}, \bar{v})$ is given by

$$
H_{Y_{y}}(\bar{u}, \bar{v})=a L^{b} \exp \left(-\frac{(180) \sqrt{\bar{u}^{2}+\overline{-}^{2}}}{\pi[c \ln (L)+d]}\right) \text {. }
$$

The chrominance spatial frequency response model $H_{C x C z}(\bar{u}, \bar{v})$ is given by

$$
H_{C x C z}(\bar{u}, \bar{v})=A \exp \left(-\alpha \sqrt{\bar{u}^{2}+\bar{v}^{2}}\right)
$$

Using the weighted least squares fitting criterion, which the parameter estimates are obtained by minimizing

$$
Q=\sum_{i=1}^{n} \lambda_{i}\left[y_{i}-f\left(\vec{x}_{i} ; \vec{\beta}\right)\right]^{2}
$$

The weights used to estimate the values of the unknown parameters are inversely proportional to the variances at each combination of predictor variable values:

$$
\lambda_{i} \propto \frac{1}{\sigma_{i}^{2}} .
$$

A circular dot-overlap model were developed for B\&W printers that accounts for the dot-overlap characteristic. In the $\mathrm{B} \& \mathrm{~W}$ case, all the dots have the same color[9]. In the color case, the overlapping segments can take different colors depending on the color of the neighboring dots.

In the proposed approach, we seek the halftone image that minimizes the weighted squared error

$$
E_{\lambda}=\sum_{k=1}^{4} \lambda_{k} \sum_{i, j}\left(z_{i, j}-w_{i, j}\right)^{2}
$$

where $Z_{i, j}=n\left(x_{i, j}\right) * h_{i, j}^{\prime}, \quad w_{i, j}=n\left(p_{i, j}\right) * h_{i, j}$ and $p_{i, j}=P\left(W_{i, j}\right)$. The $\lambda_{k} \quad(k=1,2,3,4)$ are weighting operators and $W_{i, j}$ is composed of bi,j and $*$ indicates convolution. Note that we have allowed different impulse responses $h_{i, j}, h_{i, j}^{\prime}$ for the eye filters corresponding to the halftone and continuous-tone images. The same filter is used to all the color components.

\section{Experimental Results and Discussion}

In this paper, we use the baboon color image of sizes $512 \times 512$ to demonstrate the experimental results. In the experiment, we compare two algorithms: color Lsmb and the proposed algorithms.

Firstly, we use fuzzy c-means clustering to divide several regions. The color image is partitioned into two, three and four regions using fuzzy c-means image segmentation method. Each label shows a different class. The results are shown in Fig. 1.
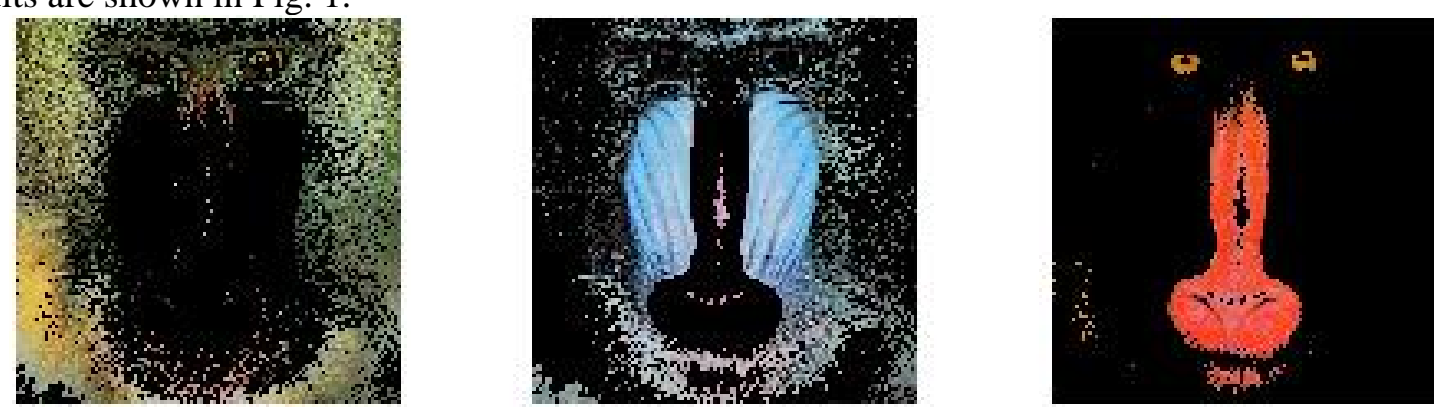

Fig. 1 The divided regions

Then we use the proposed algorithm to obtain the color halftone images. 


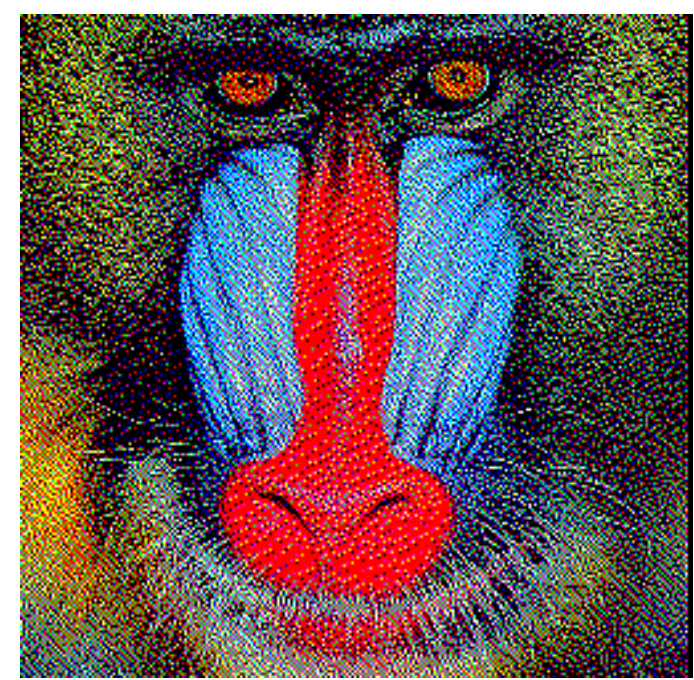

(a)

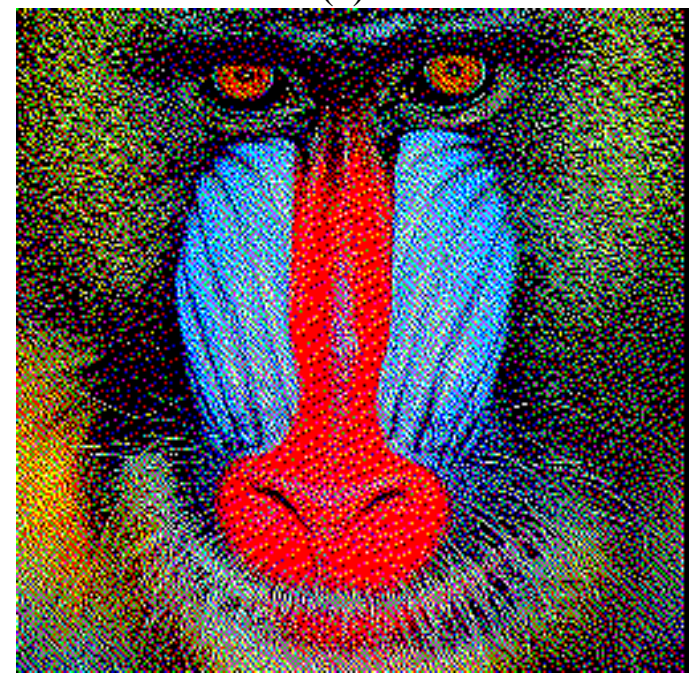

(c)

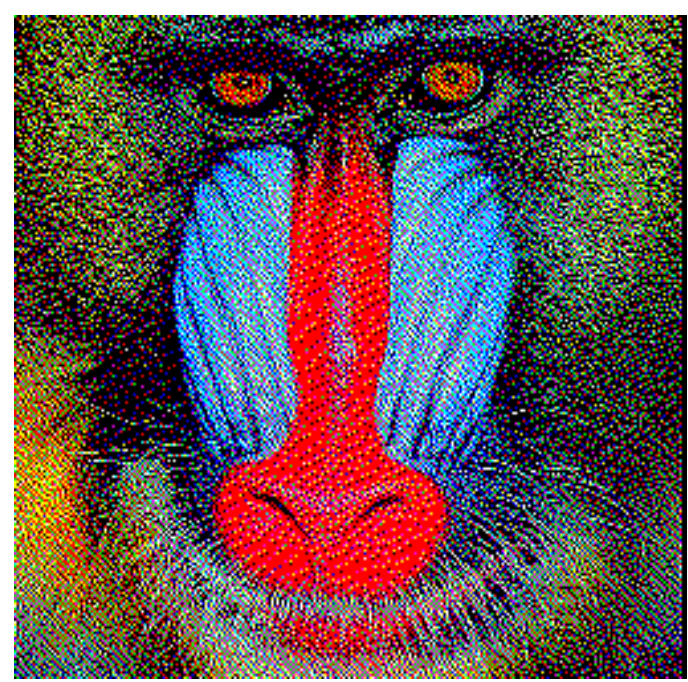

(b)

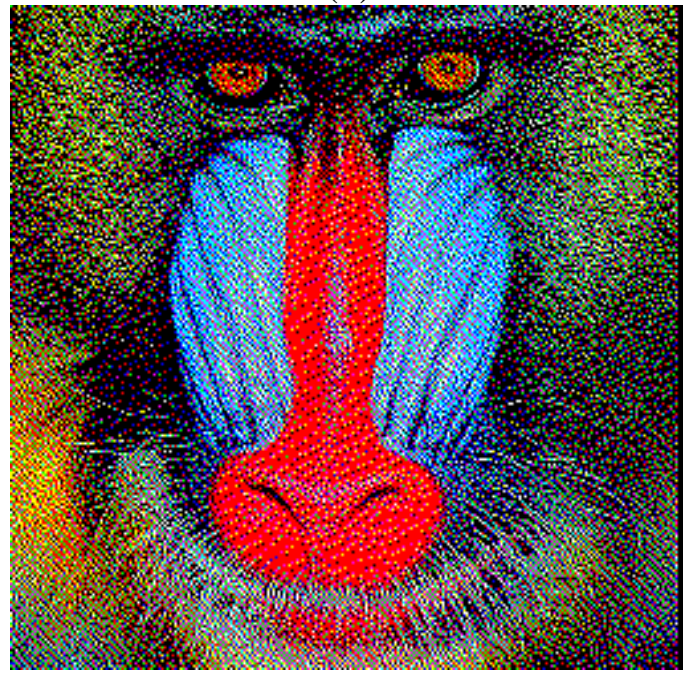

(d)

Fig. 2 Color Lsmb halftoning of proposed algorithm (a)Lsmb (b)weighed operator $\lambda_{1}, \lambda_{2}$ (c)weighed operator $\lambda_{1}, \lambda_{2}$ and $\lambda_{3}$ (d) weighed operator $\lambda_{1}, \lambda_{2}, \lambda_{3}$ and $\lambda_{4}$

Fig. 2 show the halftone of the starting point obtained is a random start. On testing with the image, we founded that when comparing the quality measurements of halftone images with MSEv and PSNR. The test results are shown in Fig. 3 .
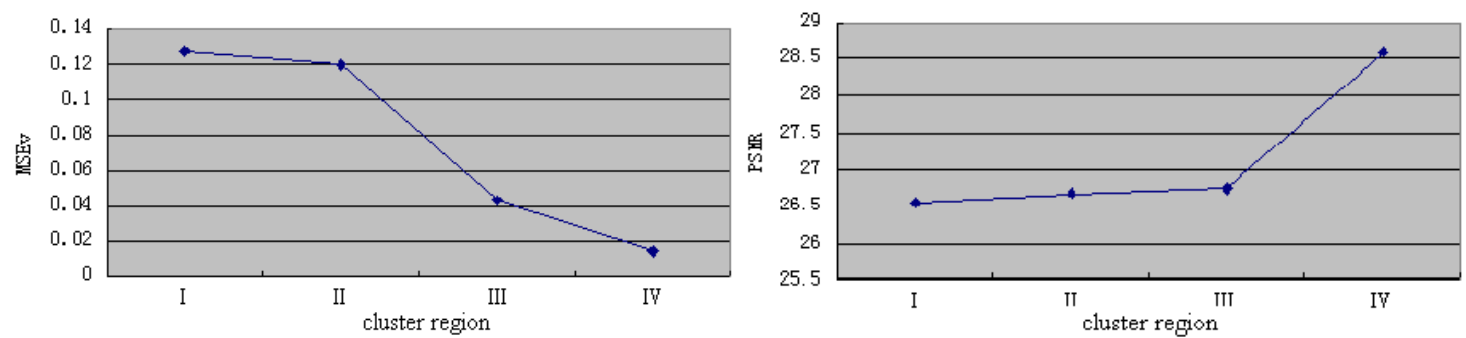

Fig. 3 Comparison of the Value of MSEv and PSNR for the Test Images

It is clear from Fig. 3 that the proposed algorithm achieves consistently lower values of MSEv than the color Lsmb algorithm, and with the increase in cluster partition, the value of MSEv is also decreases. We can be seen from Fig. 4 that the proposed algorithm achieves consistently higher values of WSNR and PSNR than the color Lsmb algorithm, and with the increase in cluster partition, the value of WSNR and PSNR are also increase. 


\section{Conclusions}

This paper developed a based on the color image content digital halftoning. A detailed analysis on the proposed algorithm was presented. Analysis results show that the proposed algorithm produces better color halftone image quality when we increase the number of clustering, in particular details of the image. Simulation results also demonstrated that the proposed algorithm achieves consistently lower values of MSEv than the color Lsmb algorithm. As the cluster partition increases, the value of MSEv decreases. And the proposed algorithm achieves consistently higher values of WSNR and PSNR than the color Lsmb algorithm. As the cluster partition increases, the value of WSNR and PSNR decreases.

\section{Acknowledgments}

This work was supported by Project 61302173 and 61461022 of the National Science Foundation of China and Foundation of Kunming University of Science and Technology under Grant 14118777, KKZ3201401003.

\section{References}

[1] R. W. Floyd and L. Steinberg: in Proc. Soc. Inf. Display, 1975, p. 36

[2] G. Sarailidis and I. Katsavounidis:IEEE Trans. Image Process., vol. 21(2012),p. 2693

[3] Liu Zhenglin Guo Xu Zou Xuecheng Xiao J ianping: J. Huazhong Univ.of Sci. \& Tech. (Nature Science Edition). 34 (5), (2006)p.69

[4] David J. Lieberman and Jan P.Allebach:IEEE Trans. Image Process. 9 (11), (2000),p.1950

[5] T. Flohr and B. Kolpatzik: in Proc. SPIE Human Vision, Visual Processing, and Digital Display IV, vol. 1913, 1993, p. 270

[6] A.Ufuk Agar, Jan P.Allebach:IEEE Trans. Image Process Vol. 14(2005) ,p. 1945

[7]Nock, R. and Nielsen, F: IEEE Trans. on Pattern Analysis and Machine Intelligence, 28 (8), p.1

[8]Bezdek, James C. Pattern Recognition with Fuzzy Objective Function Algorithms (1981).

[9] Ursula U. Müller. Metrika,2007,p.39 